\title{
SÍNDROME DE DOWN E ESQUIZOFRENIA: UM RELATO DE CASO
}

\section{DOWN SYNDROME AND SCHIZOPHRENIA: A CASE REPORT}

\section{Resumo}

A esquizofrenia e a síndrome de Down são condições prevalentes na população geral, entretanto a comorbidade entre elas é rara. Devido ao escasso material na literatura científica abrangendo tal comorbidade, é justificada a produção científica do presente trabalho. A paciente deste relato de caso tem 15 anos e apresenta síndrome de Down. Foi encaminhada ao Centro de Atenção Psicossocial Infantojuvenil pela Unidade Básica de Saúde em que fazia acompanhamento psiquiátrico. Do ponto de vista psiquiátrico, a paciente apresentava sintomas como solilóquios, alucinações auditivas e delírios, os quais foram melhorando após a introdução e aumento progressivo da olanzapina até a dose de $10 \mathrm{mg} /$ dia. Entretanto, também apresentou ao longo das avaliações sintomas de síndrome extrapiramidal (SEP), controlados com biperideno $4 \mathrm{mg} / \mathrm{dia}$. Além disso, foram constatados sintomas de ansiedade e tricotilomania, manejados com fluoxetina atéa dose de $80 \mathrm{mg} /$ dia. Emsuaúltima avaliação, a paciente apresentou-se ao serviço com melhora importante dos sintomas psicóticos, porém em alguns momentos com sintomas residuais. Assim como o relato de Buttler et al., nosso caso teve melhora dos sintomas psicóticos com baixa dose do antipsicótico, todavia o relato de Buttler et al. não descreveu a presença de SEP ou sintomatologia residual, o que dificulta a comparação. Em nossa revisão da literatura, não encontramos relatos de esquizofrenia com início precoce em pacientes com síndrome de Down e nenhuma publicação recente sobre o tema. Entretanto, o reconhecimento e o tratamento precoce da comorbidade têm potencial para um melhor prognóstico do quadro.

Palavras-chave: Esquizofrenia, síndrome de Down, comorbidade.

\section{Abstract}

Schizophrenia and Down syndrome are prevalent in the general population, however comorbidity of the two conditions is rare. Scarcity of material available in the scientific literature covering such comorbidity justifies the publication of the present report. The patient here described was 15 years old and had Down syndrome. She was referred to the Pediatric Psychosocial Care Center by the basic health unit where she received psychiatric care. From a psychiatric point of view, the patient presented symptoms such as soliloquys, auditory hallucinations and delusions, which improved after the introduction and progressive increase of olanzapine until $10 \mathrm{mg} /$ day. However, she also presented extrapyramidal symptoms, controlled with biperiden $4 \mathrm{mg} /$ day. In addition, symptoms of anxiety and trichotillomania were observed and controlled with fluoxetine up to a dose of $80 \mathrm{mg} /$ day. In her latest evaluation, the patient presented with an important improvement of psychotic symptoms, but occasional residual symptoms. As also reported by Buttler et al., our case showed an improvement of psychotic symptoms with a low dose of antipsychotics, however Buttler et al. did not describe the presence of extrapyramidal symptoms or residual symptomatology, which hinders comparison between both cases. In our review of the literature, neither previous reports of schizophrenia with early onset in patients with Down syndrome nor recent publications on the topic were found. However, the recognition and early treatment of comorbidity could potentially improve prognosis.

Keywords: Schizophrenia, Down syndrome, comorbidity. 


\section{MAIALU PEDREIRA MESSIAS', ETIENNE DE MIRANDA SILVA', LUCAS NASCIMENTO LAGO' ${ }^{1}$, LUIZ GUSTAVO MAESTRELLI', MARIELE HERTHA DE ANDRADE' ${ }^{1}$, ANDERSON SOUSA MARTINS DA SILVA²}

Residente de Psiquiatria, Secretaria Municipal de Saúde de São Bernardo do Campo, São Bernardo do Campo, SP. ${ }^{2}$ Supervisor, Residência de Psiquiatria, Secretaria Municipal de Saúde de São Bernardo do Campo, São Bernardo do Campo, SP.

\section{INTRODUÇÃO}

As prevalências de síndrome de Down (SD) e esquizofrenia são, respectivamente, 1 para $800^{1}$ e 4,6 para $1.000^{2}$. Apesar de serem prevalentes na população geral, a comorbidade é rara.

A SD é a principal causa de deficiência intelectual, acometendo quase todos os pacientes $^{3}$, que estão susceptíveis a diversas condições neurológicas e psiquiátricas, sendo as mais comuns: transtornos depressivos, transtornos ansiosos e demências precoces ${ }^{4}$. Cerca de 75\% dos pacientes com SD, aos 40 anos, apresentam sintomas de demência Alzheimer-like ${ }^{5}$.

Existem poucos relatos na literatura médica e escasso material sobreSD e esquizofrenia comórbidas ${ }^{6-9}$, justificando a necessidade de produção científica sobre o tema.

\section{RELATO dO CASO}

Paciente do sexo feminino, 15 anos, branca, natural de Ribeirão (PE), solteira, portadora de SD. Foi encaminhada pela Unidade Básica de Saúde (UBS) onde fazia acompanhamento psiquiátrico, há 8 meses, com prescrição de risperidona $2 \mathrm{mg}$ ao dia e relato de avaliação neurológica sem alterações (eletroencefalograma e tomografia de crânio normais). Segundo o encaminhamento, fez uso anterior de haloperidol (2 mg/ $\mathrm{ml}$ ) cinco gotas ao dia, com melhora parcial dos sintomas, mas devido à ocorrência de síndrome extrapiramidal (SEP), foi substituído por risperidona $2 \mathrm{mg} / \mathrm{dia}$.

Apesar do acompanhamento, foi encaminhada ao Centro de Atenção Psicossocial Infantojuvenil (CAPS IJ) por alteração de comportamento, solilóquios, hipersexualidade e perda de controle de esfíncteres.

Na entrevista inicial do CAPS IJ, a paciente apresentava dificuldade de interação com o examinador e solilóquios. Estava há 3 dias sem medicação, que a genitora suspendeu após a paciente apresentar crise oculógira por mais de 4 horas (esse sintoma regrediu após interrupção do antipsicótico). Além do quadro psiquiátrico, a genitora referiu persistência de canal arterial, nistagmo e asma em acompanhamento. Nos antecedentes familiares, havia relato de avó materna portadora de esquizofrenia. Nesse momento, foi introduzida olanzapina $5 \mathrm{mg} / \mathrm{dia}$ e realizados os seguintes diagnósticos pela Classificação Internacional de Doenças e Problemas Relacionados à Saúde, 10a edição (CID-10): F20.9 + F71 + Q90.9.
Após 7 dias, retornou ao serviço com a mãe, que relatou episódio de SEP (tremor e rigidez muscular discretos) com duração de 30 minutos, sendo por isso prescrito biperideno 2 mg/dia. Em novo retorno, houve melhora do autocuidado e da interação com os pais, porém mantinha sintomas psicóticos (alucinações auditivas e delírios). Diante do exposto, optou-se pelo aumento da olanzapina para 7,5 mg/dia e manutenção de biperideno $2 \mathrm{mg} / \mathrm{dia}$.

Após 1 mês, a criança compareceu ao serviço, e a genitora referiu melhora das alucinações auditivas, porém ainda com sintomas residuais e novos episódios de SEP. A conduta foi aumentar a dose da olanzapina para $10 \mathrm{mg} /$ dia e biperideno para $4 \mathrm{mg} / \mathrm{dia}$. Os exames laboratoriais solicitados apresentaram alteração da função tireoidiana ( TSH = 9,30; T4 livre = 1,1) e o clínico iniciou levotiroxina na dose de $25 \mathrm{mcg} / \mathrm{dia}$, posteriormente ajustada para $50 \mathrm{mcg} / \mathrm{dia}$.

Em novo retorno ao serviço, a mãe informou melhora dos sintomas psicóticos, porém ansiedade e tricotilomania. Optou-se por manter doses da olanzapina $10 \mathrm{mg} /$ dia, biperideno $4 \mathrm{mg} /$ dia e iniciar fluoxetina 20 $\mathrm{mg} / \mathrm{dia}$. Após a introdução, em um primeiro momento, os pais relataram piora da ansiedade e automutilação (escoriações em couro cabeludo), além do aumento do apetite e episódios de vômitos pós-prandiais. Optamos pelo aumento da fluoxetina para $40 \mathrm{mg} / \mathrm{dia}$, mantendose as demais medicações, então houve melhora parcial da ansiedade e dos episódios de vômito. A remissão dos sintomas ansiosos e automutilações só foi obtida com $80 \mathrm{mg} /$ dia de fluoxetina.

Apesar dos sintomas psicóticos e ansiosos controlados, a paciente apresentou aumento ponderal (ganho de $8 \mathrm{~kg}$ desde o início do tratamento) e hiperfagia. Após introdução de metformina 500 mg e ajuste para 850 $\mathrm{mg} / \mathrm{dia}$, houve melhora importante da hiperfagia e perda ponderal de $5 \mathrm{~kg}$.

Em sua última avaliação, paciente estava em uso de levotiroxina $50 \mathrm{mcg} / \mathrm{dia}$, metformina $850 \mathrm{mg} /$ dia, olanzapina $10 \mathrm{mg} / \mathrm{dia}$ e biperideno $4 \mathrm{mg} / \mathrm{dia}$. Não apresentava queixas de sintomas ansiosos, SEP ou hiperfagia. Houve melhora importante dos sintomas psicóticos, porém em alguns momentos a genitora relata que a paciente mantém solilóquios e relatos de alucinações auditivas. 


\section{Discussão}

Ao cruzarmos os Medical Subject Headings (MeSH) Down syndrome e schizophrenia, encontramos apenas dois artigos que abordam a epidemiologia dessa comorbidade e outros dois que discutem aspectos funcionais em relatos de caso sem abordar a epidemiologia.

Collacot et al. ${ }^{8}$ encontraram uma prevalência de 1,6\% da comorbidade em uma amostra com 378 adultos com SD. Porém, esse estudo utilizou a $9^{a}$ edição da CID (CID-9) e agrupou os pacientes com estados paranoides no mesmo grupo dos esquizofrênicos, fato que pode ter superestimado a prevalência. Podemos comparar com Myers et al. ${ }^{6}$, que em 1991, com uma amostra de 425 pacientes com SD, não encontraram nenhum paciente que preenchia os critérios para esquizofrenia pela CID-10.

Ainda sobre os outros estudos encontrados na busca, Cooper et al. ${ }^{9}$ publicaram uma compilação de sete casos da comorbidade de SD e esquizofrenia, onde os pacientes acometidos não se diferenciavam dos pacientes que apresentavam apenas SD no quesito funcionalidade, avaliado pela Adaptive Behavior Scale. Já Buttler et al. ${ }^{7}$ descrevem com detalhes um relato de caso de uma comorbidade de SD e esquizofrenia e as dificuldades do tratamento odontológico em um cenário rural.

Assim como o relato de Buttler et al. ${ }^{7}$, nosso caso teve melhora dos sintomas psicóticos com baixa dose do antipsicótico, porém o de Buttler et al. não relatou a presença de SEP ou sintomatologia residual, o que dificulta a comparação com o nosso caso. Os outros estudos levantados não abordam o tratamento medicamentoso e a sintomatologia residual ${ }^{6,8,9}$.

\section{CONCLUSÃO}

O risco de demência precoce em pacientes com SD é bem estabelecido ${ }^{5}$, porém em nossa revisão da literatura médica não encontramos nenhum relato de esquizofrenia com início precoce e nenhuma publicação recente sobre o tema. Como ambas as doenças têm um curso deteriorante, o reconhecimento da comorbidade e o tratamento precoce têm potencial para um melhor prognóstico do quadro.
Artigo submetido em 21/03/2019, aceito em 24/04/2019. Os autores informam não haver conflitos de interesse associados à publicação deste artigo.

Fontes de financiamento inexistentes.

Correspondência: Maialu Pedreira Messias, Rua Francisco Visentainer, 800, Assunção, CEP 09608-040, São Bernardo do Campo, SP. E-mail: maialupedreira@hotmail.com

\section{Referências}

1. Canfield MA, Honein MA, Yuskiv N, Xing J, Mai CT, Collins JS, et al. National estimates and race/ ethnic-specific variation of selected birth defects in the United States, 1999-2001. Birth Defects Res A Clin Mol Teratol. 2000;76:747-56.

2. Saha S, ChantD, Welham J, McGrath J.Asystematic review of the prevalence of schizophrenia. PLoS Med. 2005;2:e141.

3. Karmiloff-Smith A, Al-Janabi T, D'Souza H, Groet J, Massand E, Mok K, et al. The importance of understanding individual differences in Down syndrome. F1000Res. 2016 Mar 23;5. pii: F1000 Faculty Rev-389. doi: 10.12688/ f1000research.7506.1. eCollection 2016.

4. Head E, Lott IT. Down syndrome and beta-amyloid deposition. Curr Opin Neurol. 2004;17:95-100.

5. Evenhuis HM. The natural history of dementia in Down's syndrome. Arch Neurol. 1990;47:263-7.

6. Myers BA, Pueschel SM. Brief report: a case of schizophrenia in a population with Down syndrome. J Autism Dev Disord. 1994;24:95-8.

7. Butler DR, Chilvers CR, Cane RJ. The implications and management of acute odontogenic infection in association with Down and Eisenmenger syndromes and schizophrenia in a rural setting. Aust Dent J. 2007:52::61-6.

8. Collacott RA, Cooper SA, Mcgrother C. Differential rates of psychiatric disorders in adults with Down's syndrome compared with other mentally handicapped adults. $\mathrm{Br} J$ Psychiatry. 1992;161:671-4.

9. Cooper SA, Druggiral C, Collacott RA. Adaptative behavior after schizophrenia in people with Down's syndrome. J Intellect Disabil Res. 1995;39:201-4. 